\title{
Estrogen receptor acetylation and phosphorylation in hormone responses
}

\author{
C. Wang, M. Fu, R. G. Pestell \\ Lombardi Comprehensive Cancer Center, Department of Oncology, Georgetown University, \\ Washington, DC, USA.
}

\begin{abstract}
Histone acetylation is thought to facilitate binding of transcription factors (TFs) to specific target DNA sequences by destabilizing nucleosomes bound to the promoter region of a target gene. In addition, non-histone proteins including a subset of TFs and co-activators are acetylated by $\mathrm{p} 300 / \mathrm{CBP}$ and P/CAF. The regulation of estrogen signaling by direct estrogen receptor alpha $(E R \alpha)$ post-translational modification reveals a novel role for histone acetyltransferase in hormone signaling. ER $\alpha$ is acetylated and phosphorylated and phosphorylation occurs at multiple sites in response to kinase signaling. The finding that mutations with the ER $\alpha$ hinge domain lysine residues enhance hormone sensitivity suggests these residues may be involved in ligand-dependent transcriptional repression or transcriptional attenuation. Phosphorylation and acetylation of the ER regulates hormone signaling and is being assessed for a role in resistance to anti-estrogen therapy of ER $\alpha$-positive patients.
\end{abstract}

Keywords: Acetylation; Breast cancer; Estrogen receptor; Metastasis

In the USA, breast cancer accounts for approximately $30 \%$ of all cancers diagnosed and remains the second leading cause of cancer death in women. The importance of estrogen receptor alpha $(E R \alpha)$ activity in breast cancer onset is evidenced by the efficacy of $\mathrm{ER} \alpha$ antagonists for breast cancer prevention in highrisk individuals $[1,2]$. The Stathmin staging classification (TNM) includes ER $\alpha$ status [3], as the abundance of $\mathrm{ER} \alpha$ determined by immunoreactivity provides independent evidence for high survival rate and lower relapse [4,5].

Estrogens regulate cellular proliferation and differentiation of the normal breast. The two related nuclear receptors, ER $\alpha$ and $E R \beta$, function as transcription factors (TFs) to regulate downstream target genes,

Correspondence to: Richard G. Pestell, Lombardi Comprehensive Cance Center, Department of Oncology, Georgetown University, Washington, DC 20057, USA.

Received 20/05/05

Accepted 24/05/05

First published online 30/09/05

BCO/319/2004/FO co-ordinating cell-cycle progression within the mammary epithelial cell and contribute to intracrine and paracrine signaling. ERs are members of the nuclear steroid hormone receptor family, which function as ligand-dependent transcriptional regulators. The subcellular location of the ER $\alpha$ also contributes to distinct signaling pathways. Thus, in addition to the well-known nuclear function, membrane-associated $\mathrm{ER} \alpha$ also functions through membrane tyrosine kinase receptor signaling pathways [6]. Additional types of functional ERs associated with plasma membranes, such as GPR30, are thought to regulate non-genomic signaling through second messenger $\mathrm{Ca}^{2+}$, nitric oxide and protein-lipid kinases [7]. The ER $\alpha$ encodes distinct functional domains (termed A-F) conserved with other members of the classical receptor subclass. The conserved DNA-binding domain consists of 68 amino acids with two zinc finger structures. The activation function (AF) domains, $A F-1$ and $A F-2$, of the $\mathrm{ER} \alpha$ contribute synergistically to transcriptional induction. AF-1 function is induced by oncoproteins, growth factors, the co-activator p300, p68 RNA helicase $A$ and several other signaling pathways [8-10]. The 
ligand-binding domain consists of $\alpha$ helices projecting away from the hormone-binding pocket in the absence of ligand. In the presence of ligand, the ER $\alpha$ and its carboxyl terminal helix 12 undergo conformational change, creating new structural surfaces that alter binding of co-repressors and recruit co-activators, promoting transcriptional activity.

The function of ER $\alpha$ is regulated by posttranslational modification and functional interaction in trans with an array of distinct proteins. Post translational modification of the nuclear receptors co-ordinate distinct functional activities. ER $\alpha$ is phosphorylated by distinct kinases, including extracellular-regulated kinases (ERK) 1/2, p38 MAP kinase (MAPK), cyclin A/cdk2, cdk7, c-Src, pp90-RSK1, AKT, IKK $\alpha$ and protein kinase $A$. $E R \alpha$ forms multiprotein complexes with co-activator and co-repressor proteins. These complexes encode multiple distinct enzyme activities, including histone acetyl transferase (HAT), histone methyl transferases and chromatin remodeling proteins. The nuclear receptor co-activators of the p160 family (SRC1, SRC2, SRC3) interact with the nuclear receptor in a ligand-regulated manner through the AF-2 domain. $\alpha$-Helical motifs within the p160 proteins are required for interaction with nuclear receptors. ER $\alpha$ co-activators include P/CAF, p68 RNA helicase, p300/CBP, BRG1, the SRC family, TIP60 and others (reviewed in [11]). It has been hypothesized, as growth factor stimulation regulates many of these mitogenic signaling kinases, that phosphorylation of the ER $\alpha$ may contribute to proliferative signaling and/or clinical resistance to anti-estrogen therapies, such as tamoxifen.

The co-elution of ER $\alpha$ with the HATs prompted biochemical studies which identified the $\mathrm{ER} \alpha$ as a bona fide substrate for p300 [12]. Acetylation of histones involves the transfer of an acetyl group from acetyl co-enzyme A (acetyl CoA) to the $\varepsilon$-amino group of lysine side chains. This reaction is conducted by a growing family of HATs, including p300/CBP and SRCs, which have distinct substrate specificities [13]. Acetylation is now known to regulate the function of many proteins, including histones, structural proteins, cell-cycle control protein, TFs and the HAT enzymes themselves [11,14]. Histone acetylation leads to local changes in electrostatic interactions, changes $\alpha$-helical content of histone tails and creates recognition sites to recruit other TFs and co-regulatory proteins. The sequential recruitment of other proteins forms the basis of local signaling modules within the chromatin of the histone tails, referred to as the 'histone code hypothesis' [15]. A growing list of nonhistone substrates for HATs, known as Factor Acetyl Transferase Substrates (FATS), underscores the broad importance of this modification in biology (Fig. 1).

Recent studies have demonstrated the $\mathrm{ER} \alpha$ is acetylated in breast cancer cells [12]. The ER $\alpha$ acetylation site was identified through MALDI-TOF

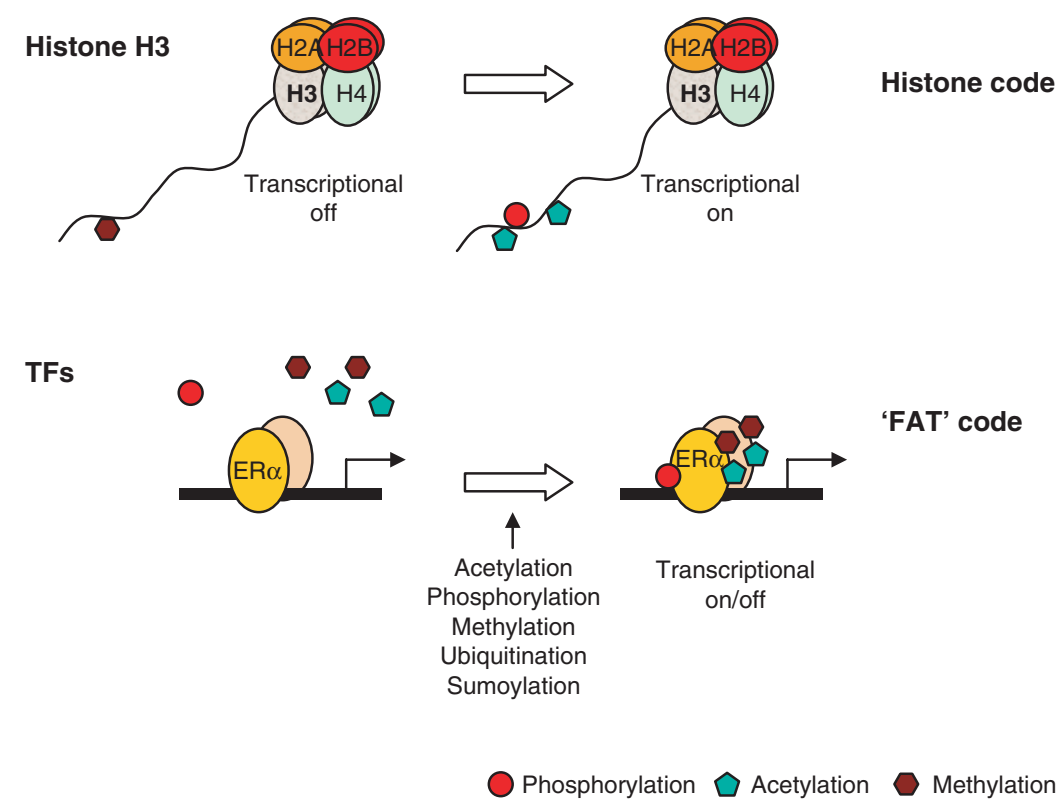

Figure 1.

A schematic representation of a nucleosome core particle, consisting of a histone octamer wrapped in DNA. The histone tail is shown extending to the left of core histones. The core histone tails contain motifs that can be post-translationally modified by phosphorylation, acetylation and methylation. Below TFs are shown bound to DNA. Post-translational modification of TFs by acetylation in turn integrates intramolecular signaling by phosphorylation, methylation and other posttranslational modifications. The intramolecular signal between phosphorylation, acetylation and other post-translational modifications are referred to as the FAT code. 
mass spectrometry and sequencing by Edman degradation. The acetylated residues (lysines 299, 302, 303) are located within the ER $\alpha$ hinge region, predicted to reside in proximity to the hydrophobic ligandbinding pocket. Although the ER $\alpha$ binds several HATs, including P/CAF, ER $\alpha$ is preferentially acetylated by p300, and lysines 302 and 303 were preferentially acetylated, suggesting an important selectivity in substrate recognition [12]. Mutational analysis of the ER $\alpha$ lysine residues demonstrated a role in basal-level and ligand-regulated activity. Substitution of the lysine residues enhanced activation of the ER $\alpha$ at low concentrations of estradiol $[12,16]$.

Post-translational modification by acetylation and phosphorylation are integrated in several physiological circumstances. Histone acetylation and phosphorylation occurs contemporaneously upon induction of immediate early-gene induction. Histone $\mathrm{H} 3$ is both acetylated and phosphorylated [17]. Mutation of the androgen receptor (AR) acetylation site reduced CAMP and AKT but not MAPK signaling $[18,19]$. The $A R$ acetylation site affected AR phosphorylation. Mutation of each of the six AR phosphorylation sites demonstrated a single phosphorylation site, Ser94, regulated the AR response to histone deacetylation. Together, these studies suggested the AR lysine residues may co-ordinate the function of a subset of kinase modules signaling to the AR. To assess the possibility that phosphorylation and acetylation site events were interdependent in regulating $\mathrm{ER} \alpha$ activity, growth factor regulation of $\mathrm{ER} \alpha$ activity was examined. AF-1 functions, including growth factor-induced, ligand-independent $\mathrm{ER} \alpha$ activity, mediated through MAPK, and activation by p68 RNA helicase A, were not affected by $E R \alpha$ acetylation [12]. However, mutation of the PKA phosphorylation site at residue 305, to mimic constitutive phosphorylation, blocked in vivo acetylation of the $\mathrm{ER} \alpha$ at $\mathrm{K} 303$, suggesting these two post-translational modifications are coupled within the ER $\alpha$ receptor [20]. Resistance to the antiestrogen tamoxifen involves PKA signals to ER $\alpha$ S305 [21]. cAMP-dependent phosphorylation site mutants of this residue enhanced hormone sensitivity of the $\mathrm{ER} \alpha$ and inhibited ER $\alpha$ acetylation [20]. Together, these studies implicate the ER $\alpha$ lysine motif in an intramolecular signaling response in tamoxifen.

The $E R \alpha$-acetylated lysine motif is conserved with many other nuclear receptors [12]. Subsequent studies have confirmed this motif is a site of acetylation in other receptors, which regulates nuclear receptor function in a profound manner $[13,18,19,22]$. The AR is acetylated by several HATs including P/CAF, p300 and TIP60 [18,23,24]. Functional analysis has provided evidence for TF acetylation in growth control. Generation of AR acetylation mimic mutants revealed a role for TF acetylation in growth control [18,23,24].
Since acetylation of lysine residues can neutralize their positive charge and increase their hydrophobicity, AR mutants encoding lysine residues substituted with polar uncharged or polar residues were generated as acetylation mimic mutants. Comparison was made with mutants encoding charged residue to create 'dead' mutants. AR acetylation mimic mutants enhanced binding to co-activator proteins (p300) and reduced binding to co-repressors (HDAC/NCoR/ Smad3). The acetylation mimic AR mutants conveyed enhanced prostate cancer cellular growth in soft agar and in nude mice. The acetylation mimic mutants were resistant to the effects of the androgen antagonist flutamide [18,24]. The $E R \alpha$ acetylation site also appears to govern co-regulator recruitment and cellular growth. MCF7 cells expressing ER $\alpha$ K303R show enhanced proliferation in response to ligand and increased binding to SRC2 [16].

Based on studies examining acetylation of histone and the AR, several predictions can be made for $E R \alpha$ acetylation. AR acetylation mimic mutants are defective in repression by and recruitment of HDAC/NCoRcontaining complexes. Several ER $\alpha$ co-repressors contain histone deacetylases. The metastasis-associated protein family (MTA) members, MTA1 and MTA2 for example, are found within nucleosome remodeling and histone deacetylation (NuRD) complexes, and BRCA1 is co-associated with HDACs [25] (Fig. 1). BRCA1 and MTA1 inhibit ER $\alpha$ signals. It would be predicted that the $\mathrm{ER} \alpha$ acetylation site may convey resistance to BRCA1 or MTA repression. If confirmed, such findings would provide a key link between breast tumor suppression and estrogen hormone signaling. Acetylation of nuclear receptors occurs through a conserved motif. In addition, functional properties of nuclear receptors, including tumor growth in vivo, are regulated by acetylation. Therefore, receptor acetylation is being assessed as a new target for cancer therapies [26].

\section{Acknowledgements}

This work was supported in part by awards from the Susan G. Komen Breast Cancer Foundation, BCTR0504227 (C.W.), R01CA70896, R01CA75503, R01CA86072, R01CA93596, R01CA107382 (R.G.P.) and NIH 1 R21DK065220-02 (NIDDK) (M.F.). Work conducted at the Lombardi Comprehensive Cancer Center was supported by the NIH Comprehensive Cancer Center Core grant (CA51008-13) (R.G.P.).

\section{References}

1. Jordan VC. Designer estrogens. Sci Am 1998; 279: 60-67.

2. Dickson RB, Pestell RG, Lippman M. Molecular biology of breast cancer. In: Devita V, Jellman S, Rosenberg S (Eds). 
Cancer: Principles and Practice of Oncology, 7th edition. Philadelphia, PA: JB Lippincott; 2005: 1399-1414.

3. Fitzgibbons PL, Henson DE, Hutter RVP. Benign breast changes and risk for subsequent breast cancer: an update of the 1985 consensus statement. Cancer Committee of the College of American Pathologists. Arch Pathol Lab Med 1998; 122: 1053-1055.

4. Brodie A. Aromatase inhibitor development and hormone therapy: a perspective. Semin Oncol 2003; 30(Suppl 14): $12-22$.

5. Jordan VC, Morrow M. Tamoxifen, raloxifene, and the prevention of breast cancer. Endocr Rev 1999; 20: 253-278.

6. Migliaccio A, DiDomenico M, Castoria G, et al. Tyrosine kinase/p21ras/MAP-kinase pathway activation by estradiol-receptor complex in MCF-7 cells. EMBO $J$ 1996; 15: 1292-1300.

7. Revankar CM, Cimino DF, Sklar LA, et al. A transmembrane intracellular estrogen receptor mediates rapid cell signaling. Science 2005; 307: 1625-1630.

8. Yue W, Wang JP, Conaway MR, et al. Adaptive hypersensitivity following long-term estrogen deprivation: involvement of multiple signal pathways. J Steroid Biochem Mol Biol 2003; 86: 265-274.

9. Kato $\mathrm{S}$, Endoh $\mathrm{H}$, Masuhiro $\mathrm{Y}$, et al. Activation of the estrogen receptor through phosphorylation by mitogenactivated protein kinase. Science 1995; 270: 1491-1494.

10. Kobayashi Y, Kitamoto T, Masuhiro Y, et al. p300 mediates functional synergism between AF-1 and AF-2 of estrogen receptor and by interacting directly with the $\mathrm{N}$-terminal A/B domains. J Biol Chem 2000; 275: 15645-15651.

11. Fu M, Wang $C$, Zhang $X$, Pestell R. Nuclear receptor modifications and endocrine cell proliferation. J Steroid Biochem Mol Biol 2003; 85: 133-138.

12. Wang C, Fu M, Angeletti R, et al. Direct acetylation of the estrogen receptor $\alpha$ hinge region by p300 regulates transactivation and hormone sensitivity. J Biol Chem 2001; 276: 18375-18383.

13. Fu M, Wang C, Zhang X, Pestell RG. Acetylation of nuclear receptors in cellular growth and apoptosis. Biochem Pharmacol 2004; 68: 1199-1208.

14. Bouras T, Fu M, Sauve AA, et al. SIRT1 deacetylation and repression of P300 involves lysine residues 1020/1024 within the cell-cycle regulatory domain 1 . $\mathrm{J} \mathrm{Biol} \mathrm{Chem}$ 2005; 280: 10264-10276.
15. Strahl BD, Ohba R, Cook RG, Allis CD. Methylation of histone $\mathrm{H} 3$ at lysine 4 is highly conserved and correlates with transcriptionally active nuclei in tetrahymena. Proc Natl Acad Sci USA 1999; 96: 14967-14972.

16. Fuqua SA, Wiltschke C, Zhang QX, et al. A hypersensitive estrogen receptor-alpha mutation in premalignant breast lesions. Cancer Res 2000; 60: 4026-4029.

17. Fu M, Wang C, Wang J, et al. Acetylation in hormone signaling and the cell-cycle. Cytokine Growth Factor Rev 2002; 13: 259-276.

18. Fu M, Wang C, Wang J, et al. The androgen receptor acetylation governs transactivation and MEKK1-induced apoptosis without affecting in vitro sumoylation and transrepression function. Mol Cell Biol 2002; 22: 3373-3388.

19. Fu M, Rao M, Wu K, et al. The androgen receptor acetylation site regulates $\mathrm{CAMP}$ and AKT but not ERK-induced activity. J Biol Chem 2004; 279: 29436-29449.

20. Cui $\mathrm{Y}$, Zhang M, Pestell R, et al. Phosphorylation of estrogen receptor alpha blocks its acetylation and regulates estrogen sensitivity. Cancer Res 2004; 64: 9199-9208.

21. Michalides R, Griekspoor A, Balkenende A, et al. Tamoxifen resistance by a conformational arrest of the estrogen receptor alpha after PKA activation in breast cancer. Cancer Cell 2004; 5: 597-605.

22. Gaughan L, Logan IR, Cook S, et al. Tip60 and histone deacetylase 1 regulate androgen receptor activity through changes to the acetylation Status of the Receptor. J Biol Chem 2002; 277: 25904-25913.

23. Fu M, Wang C, Reutens AT, et al. p300 and p300/ cAMP-response element-binding protein-associated factor acetylate the androgen receptor at sites governing hormone-dependent transactivation. J Biol Chem 2000; 275: 20853-20860.

24. Fu M, Wang C, Wang J, et al. Acetylation of the androgen receptor enhances coactivator binding and promotes prostate cancer cell growth. Mol Cell Biol 2003; 23 : 8563-8575.

25. Rosen EM, Fan S, Pestell RG, Goldberg ID. BRCA1 gene in breast cancer. J Cell Physiol 2003; 196: 19-41.

26. Liu MC, Marshall JL, Pestell RG. Novel strategies in cancer therapeutics: targeting enzymes involved in cell cycle regulation and cellular proliferation. Curr Cancer Drug Target 2004; 4: 403-424. 